\title{
The nature of NaSt1 from Keck spectroscopy
}

\author{
Paul A. Crowther and Linda J. Smith \\ Department of Physics \& Astronomy, UCL, Gower Street, London, UK
}

\section{What is NaSt1?}

The availability of $8-10 \mathrm{~m}$ telescopes now permit the routine observation of faint, heavily reddened peculiar emission line objects, which may provide new information on stellar evolution. One such object is NaSt1 (WR 122, IRAS 18497+0056), discovered by Nassau \& Stephenson (1963) who proposed a WR classification because of its strong emission line spectrum. Massey \& Conti (1983) identified NaSt1 as a peculiar WN10 star. However, subsequent work has argued against a genuine WR identification, favouring either $\mathrm{B}[\mathrm{e}], \mathrm{O}[\mathrm{e}]$ or Ofpe/WN9 spectral types from optical and IR spectroscopy (e.g., van der Hucht et al. 1997). NaSt1 has recently received renewed attention, principally because it is extremely bright in the IR $(K=6.5 \mathrm{mag})$. Indeed, NaSt1 is currently used as a late WN-type IR spectral standard, despite its uncertain nature.

\section{Nebular properties}

We have used the $10 \mathrm{~m}$ Keck I (+HIRES), 4.2m WHT (+ISIS, AUX) and $3.8 \mathrm{~m}$ UKIRT (+CGS4) telescopes to obtain high quality spectroscopy and imaging of NaSt1. These reveal a faint, reddened continuum ( $v=15.2 \mathrm{mag}, b-v=1.7 \mathrm{mag})$, together with a multitude of strong, narrow exclusively nebular features, including permitted and forbidden lines, covering a wide range in excitation (e.g., H I, He I-II, N I-III, [N II], [S II-III], [Ar III-V], [Ca V-VII], [Fe II-VII]). Nebular line strengths are very peculiar, with He I-II and [N II] very strong, and [O II-III] weak/absent.

We obtain $E_{B-V} \simeq 2.1 \mathrm{mag}$ towards NaSt1, with its dereddened spectral energy distribution well characterised by a hot $(\sim 30-200 \mathrm{kK})$ black body. Additional black bodies are required to reproduce the near-IR $(\sim 2000 \mathrm{~K})$ and mid-IR $(\sim 700 \mathrm{~K})$ flux distributions, indicating the presence of re-radiated warm dust. The IR colours of NaSt1 are extremely unusual. The nebular and interstellar spectral features have been used to derive a distance of $1-3 \mathrm{kpc}$ from the Brand \& Blitz (1993) Galactic rotation-curve. Assuming a realistic bolometric correction of -3 to $-6 \mathrm{mag}, \mathrm{NaSt} 1$ is intrinsically luminous $\left(\log \left(L / \mathrm{L}_{\odot}\right)=5 \pm 1\right)$.

Narrow-band WHT imaging reveals an elliptically shaped nebula in [N II] $\lambda 6584$ with an average diameter of $6 . " 8$, which corresponds to $0.03-0.11 \mathrm{pc}$, with a dynamical timescale of several thousand years. The Keck-HIRES spectroscopy indicates that the majority of optical line-profiles are double-peaked, suggestive of outflowing material, possibly with a disk-like geometry. We find a broad correlation of FWHM (in the range $\sim 30-140 \mathrm{~km} \mathrm{~s}^{-1}$ ) versus ionization potential for representative ions suggesting lines from different excitation are formed within different regions of the nebula, also known for symbiotic novae. Nevertheless, 
at representative values of $T_{\mathrm{e}} \simeq 13000 \mathrm{~K}$ and $n_{\mathrm{e}} \simeq 3 \times 10^{6} \mathrm{~cm}^{-3}$ obtained from diagnostic line-ratios, we obtain chemical abundances such that the enrichment in helium is unprecedented. Nitrogen is highly enriched, while oxygen is severely depleted. These quantities are in good agreement with CNO-processed material.

\section{A helium symbiotic nova or WR hidden by a dense disk/nebula?}

We find that the observed properties of NaSt1 do not favour a normal WR, Ofpe/WN9, B[e] or symbiotic nature, although several characteristics are shared with each class. Consequently, we shall consider two alternative possibilities.

Spectroscopy reveals many similarities with symbiotic novae, which consist of a red giant, the ionized nebula and a hot ionizing source. However, X-rays expected from the compact source are absent, as is evidence for a red giant companion and the characteristic O VI Raman scattered lines. The peculiar chemistry for NaSt1 suggests a hydrogen-stripped AGB star as a secondary, provided that the distance is $\leq 1 \mathrm{kpc}$.

Despite the absence of a stellar signature in NaSt1, our nebular analysis suggests that it contains a hot, luminous, evolved star, hidden from direct view. The most likely potential ionizing source is an early WN star. How could its stellar features not be directly observed? Perhaps its stellar wind is invisible because of either a dense accretion disk around the system, or a dense nebula ejected from the immediate progenitor of the WN star. The most plausible cause of such a nebula would be a massive eruption during its LBV phase, reminiscent of $\eta$ Carinae. The IR dust properties of NaSt1 most closely resemble $\eta$ Car. One clue may be the unusual He I $\lambda 2.0581 \mu \mathrm{m}$ profile showing wings extending to $\sim 300 \mathrm{~km} \mathrm{~s}^{-1}$, reminiscent of IR profiles in massive YSO's. Whatever its true nature, NaSt1 should no longer be considered as a late-WN classification standard in the near-IR. Our results are discussed in a forthcoming paper (Crowther \&. Smith 1999).

Acknowledgments. Thanks to Mike Barlow, Bruce Bohannan, You-Hua Chu, Steve Fossey, Marten van Kerkwijk, Mario Livio, Xiao Wei Lui, Andy Pollock, Hans-Martin Schmid, Lindsey Smith and Peter Tamblyn. The W.M. Keck Telescope is operated by Caltech and the University of Calafornia. UKIRT is operated by the Royal Observatories on behalf of the PPARC. The WHT is operated by the Royal Observatories in the Spanish Observatorio del Roque de los Muchachos of the Instituto de Astrofisica de Canarias. PAC is a Royal Society Research Fellow.

\section{References}

Brand, J., Blitz, L. 1993, A\&A 275, 67

Crowther, P.A., Smith, L.J. 1999, MNRAS in press

van der Hucht, K.A., Williams, P.M., Morris, P.W., van Genderen, A.M. 1997, in: A. Nota \& H. Lamers (eds.), Luminous Blue Variables: Massive Stars in Transition, ASP-CS 120, 211

Massey, P., Conti, P.S. 1983, PASP 95, 440

Nassau, J.J., Stephenson, C.B. 1963, Luminous Stars in the Northern Milky Way, IV (Hamburg-Bergedorf) 